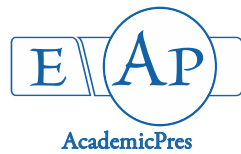

\title{
Middle East Respiratory Syndrome: Its Occurrence, Aetiology, Epidemiology, Associated Clinicopathological Findings, Diagnosis, Prevention and Control in Humans and Animals: An Overview
}

\author{
Iniobong C. I. UGOCHUKWU ${ }^{1}$, Onyinyechukwu A. AGINA ${ }^{1 *}$, \\ Emmanuel I. UGOCHUKWU² \\ ${ }^{1}$ University of Nigeria, Department of Veterinary Pathology and Microbiology, 410001 Nsukka., Enugu State, \\ Nigeria; iniobong.ugochukwu@unn.edu.ng; onyinye.noel@unn.edu.ng(*correspondingauthor) \\ ${ }^{2}$ University of Nigeria, Department of Veterinary Medicine, 410001 Nsukka. Enugu State, Nigeria; emmanuel.ugochukwu@unn.edu.ng
}

\begin{abstract}
Middle East respiratory syndrome (MERS) is a serious zoonotic disease. It is characterized by severe infection of the lower respiratory pathway in humans. Dromedary camels are considered to be the likeliest source of the very pathogenic aetiologic agent, Middle East respiratory syndrome coronavirus (MERS-CoV). Although, the first case of MERS-CoV was initially identified in Kingdom of Saudi Arabia (KSA) in September 2012, most of the reported cases have been detected in Arabian Peninsula but worthy of note is that travel-associated MERS cases have been reported around the world especially in Europe, Asia, Africa and North-America. This virus is very endemic in camel populations of East Africa and the Arabian Peninsula but worryingly, the zoonotic transmission of MERS- $\mathrm{CoV}$ are now well reported. It is diagnosed in camels using IgG immunofluorescence assay, protein microarray and virus neutralisation assay Diagnosis in humans is based on chest radiographs and computed tomographic scans but confirmatory diagnosis of suspected MERS patients could be done by taking a intratracheal aspirate for detection test of MERS-CoV RNA positive real-time reverse transcriptase polymerase chain reaction assays. The major control measures of MERS-CoV spread include strict regulation of camel movement, regular herd screening and culling of infected camels.
\end{abstract}

Keywords: camels; coronavirus; humans; Middle East Respiratory Syndrome; zoonosis

\section{Introduction}

Middle East respiratory syndrome coronavirus (MERS$\mathrm{CoV}$ ) causes severe lower respiratory tract infection in humans (Haagmans et al., 2014). MERS-CoV was first discovered in September 2012 in a patient from Saudi Arabia who had hypoxemic respiratory failure and multiorgan illness (Zaki et al., 2012). Cases of MERS-CoV infection continue to occur, including sporadic zoonotic infections in humans across the Arabian Peninsula, occasional importations and outbreaks of human-to-human transmission in health care facilities (Arabi et al., 2017).
The advent of the Middle East respiratory syndrome coronavirus (MERS-CoV) in 2012 marked the second time that a new, highly pathogenic coronavirus has emerged in the human population in the $21^{\text {st }}$ century (van Doremalen and Munster, 2015). Dromedary camels are considered to be the most likely source of zoonotic transmission. MERS$\mathrm{CoV}$ attacks the cells of the host after binding the dipeptidyl peptidase 4 (DPP-4) receptor and the carcinoembryonic antigen-related cell-adhesion molecule 5 (CEACAM5) cofactor ligand, and it replicates efficiently in the respiratory pathway epithelial cells of humans. Onset of malady begins after a period of 2 to 14 days and most times leading to respiratory failure with affected patients needing multiple organ support. Two MERS-CoV lineages have been identified in Nigerian camels although they were found to be genetically distinct from those found in camels and humans in the Middle East (Younan et al., 2016) and considering the increasing camel trade around West Africa Saharan belt, it is important to write this review. 


\section{Aetiology}

MERS-CoV is the sixth coronavirus and the first enzootic betacoronavirus of the $\mathrm{C}$ phylogenetic lineage known to affect humans (de Groot et al., 2013; Omrani et al., 2015). Lineage A includes $\mathrm{HCoV}-\mathrm{OC} 43$ and $\mathrm{HCoV}$ $\mathrm{HKU} 1$, and lineage $\mathrm{B}$ includes the severe acute respiratory syndrome (SARS) coronavirus (SARS-CoV) (Van Boheemen et al., 2012). MERS-CoV is an enveloped, singlestranded positive RNA genome and replicates in the cytoplasm (Van Boheemen et al., 2012).

MERS-CoV genomes are phylogenetically classified into 2 clades namely clade A and B (Cotton et al., 2013). The proximate source of human infection remains unclear. MERS-CoV is related to, but not identical to, viruses detected in bats (Annan et al., 2013; Ithete et al., 2013). Although, needing confirmation, a short RNA fragment of the viral polymerase region identical to MERS-CoV has been identified in Taphozous perforates bats (Memish et al., 2013a). Phylogenetic and sequencing data suggest that MERS-CoV originated from bat ancestors but after undergoing a recombination, possibly in dromedary camels in Africa, before its exportation to the Arabian Peninsula along the camel trading routes (Omrani et al., 2015).

\section{Epidemiology}

Results from archived camel sera provided evidence for circulation of MERS-CoV among dromedary camels in East Africa as far back as 1983 and in Saudi Arabia in 1992 (Younan et al., 2016). MERS-CoV was initially identified in a fatal case of severe respiratory illness in the Kingdom of Saudi Arabia (KSA) in September, 2012, and earlier cases were retrospectively identified from an outbreak of severe respiratory illness in Jordan in 2012 (Hilgenfeld and Peiris, 2013; WHO, 2015).

The majority of cases have been detected in KSA and to a lesser extent the United Arab Emirates, Qatar and Jordan (WHO, 2015). Travel-associated MERS cases have been reported in Europe, Asia, Africa and North-America. Most recently, with the travel-associated MERS case in South Korea resulted subsequently in a hospital-associated outbreak involving more than 180 cases (WHO, 2015). Fatality rate of $35 \%$ has been reported by World Health Organization (WHO, 2017). Approximately, 80\% of reported cases have been linked to exposure in KSA. Zoonotic transmission of MERS-CoV between dromedary camels and humans has also been documented in several countries. Human-to-human transmission in health care facilities accounts for most of reported cases till date, although human-to-human transmission in household settings has also been reported (Arabi et al., 2017). Epidemiologic findings suggest that human-to-human transmission occurs primarily through close contact with a patient with confirmed MERS, most likely through respiratory droplets. The risk of transmission appears to be greater during the performance of aerosol generating procedures without adequate personal protection or ventilation (WHO, 2015). Human-to-human transmission appears to be infrequent outside health care settings but has been documented after close contact with infected persons (Memish et al., 2013b). Contamination of environmental surfaces in patient rooms with MERS-CoV may result in transmission (Bin et al., 2016; Kim et al., 2016).

\section{Zoonosis}

The disease is highly endemic in camel populations of East Africa and the Arabian Peninsula. It is unclear as to when the virus was introduced to dromedary camels, but data from epidemiological studies suggests presence of the virus in dromedary camels as back as 1983. Bats and alpacas can also serve as reservoirs for MERS-CoV, but the transmission to humans is mainly through dromedary camels (Mohd et al., 2016; Younan et al., 2016) and direct exposure to dromedaries has been documented in up to $40 \%$ of primary cases (Muller et al., 2015).

Sero-epidemiologic studies have shown that antibodies to MERS-CoV are present and has circulated among in dromedary camels throughout the Middle East and Africa for at least three decades (Azhar et al., 2014). Although MERS-CoV RNA has been detected in respiratory and other body secretions, particularly among young dromedary camel however, infections in dromedaries may cause only mild clinical manifestations of the upper respiratory tract (Azhar et al., 2014). However, routes of transmission remain unclear, but contact with infectious nasal or other bodily secretions and possibly the consumption of raw dromedary products such as unpasteurized camel milk can transmit the infectious agent (Alraddadi et al., 2016). In addition, sero-epidemiologic studies indicate that infection may be associated with occupational exposures to dromedaries (Alraddadi et al., 2016). This is shown by a study in Saudi Arabia by Alraddadi et al. (2016), which showed that the rate of MERS-CoV seropositivity was 15 times more in shepherds and 23 times as high in slaughterhouse workers than in the general population.

\section{Host risk factors}

The majority of severe MERS cases have occurred in humans older than 50 years of age who have concurrent conditions such as diabetes, cardiovascular diseases, obesity, chronic respiratory disease, chronic renal disease, neoplastic conditions or in persons receiving immunosuppressive therapy (Korea Centers for Disease Control and Prevention 2016). Old age and chronic respiratory illness particularly have been associated with death among infected patients (Korea Center for Disease Control and Prevention, 2016). Mild and asymptomatic infections have occurred predominantly among young and healthy persons, including health care workers (Oboho et al., 2015; Korea Center for Disease Control and Prevention, 2016). Clinically recognized infections among children are uncommon (Memish et al., 2014). Up till date, host genetic risk factors have not been reported (Arabi et al., 2017). 
322

\section{Clinical signs associated with MERS}

The clinical picture of MERS-CoV includes asymptomatic infections, mild or moderately symptomatic cases and fatal disease (Oboho et al., 2015; Al-Tawfiq and Memish, 2016). Human patients with symptomatic MERS show clinical manifestations after a 2-14 days incubation period. Mildly symptomatic cases can be manifested by lowgrade pyrexia, rhinorrhoea, sore throat and myalgia (Arabi $e t$ al., 2014). Severe disease is characterized by acute respiratory distress syndrome (Arabi et al., 2014). In very ill patients, chest radiographs and computed tomographic scans often show multilobar alveolar disease and occasional pleural effusions (Arabi et al., 2014). Extrapulmonary manifestations are common among severely ill patients, this includes gastrointestinal symptoms, such as nausea, emesis, or diarrhoea and acute kidney injury has also been reported (Arabi et al., 2014). Stillbirth has been reported in a pregnant woman with probable MERS (Payne et al., 2014). Neurologic manifestations suggestive of encephalitis have also been reported in a few cases (Arabi et al., 2015). Haematological findings include leukopenia and lymphopenia and occasionally include thrombocytopenia and anaemia. Mild-to-moderate elevation of aminotransferase levels is common, especially among critically ill patients (Arabi et al., 2017).

\section{Pathological (gross, histopathology and clinical pathology) findings associated with MERS in humans}

Insights into the gross and histopathology of MERS are limited. The principal post-mortem finding was diffuse alveolar damage. Immunoassays showed MERS-CoV antigen in pneumocytes and epithelial syncytial cells. No evidence of extrapulmonary MERS-CoV antigen was detected, even in the kidney, this explains the fact that renal insufficiency is a common clinical finding among critically ill patients ( $\mathrm{Ng}$ et al., 2016). Human patients with MERS$\mathrm{CoV}$ are more likely to have monocytosis with normal white blood cell count and lower C-reactive protein level (Park et al., 2017). Lymphopenia and thrombocytopenia and transient leukopenia are prominent clinicopathological features of the disease, and there were no significant changes in the serum liver enzymes and creatinine concentration (Al-Tafwiq et al., 2017). Pathological (gross and histopathology) findings associated with MERS in animals

Animals ranging from mice to rabbits to non-human primates have been inoculated with MERS-CoV, with varying outcomes. Mice were susceptible to infection and developed severe disease. Rhesus macaques and marmosets are also susceptible, but marmosets developed more severe disease (van Doremalen and Munster, 2015). MERS-CoV affects primarily camel and some of these dromedary camels show respiratory symptoms (Omrani et al., 2015). In rabbits experimentally inoculated with MERS-CoV, the following histopathologic findings were observed- focal mild to moderate rhinitis with heterophils in the epithelium and lamina propria, focal mild to moderate necrosis and epithelial hyperplasia and regenerational hypertrophy were observed in the nasal cavity of MERS-CoV-inoculated animals (Haagmans et al., 2015). There were mild thickening of the terminal bronchioles and the interalveolar septa with increased numbers of heterophils. There was also mild hypertrophy of type II pneumocytes and moderate proliferation of the bronchus-associated lymphoid tissue (BALT) (Haagmans et al., 2015).

In Rhesus Macaques, the pneumonic lesions were characterized as multifocal, mild to marked interstitial pneumonia, characterized by thickening of alveolar septae by oedematous fluid and fibrin; there were also the presence of alveolar macrophages and neutrophils (de Wit et al., 2013). Many of these alveolar macrophages formed multinuclei syncytia similar to those described as bronchiolitis obliterans organizing pneumonia. This organizing pneumonia consisted of aggregations of fibrin, macrophages, and sloughed pulmonary epithelium that fill and occlude small airways. Also, early stage lesions of bronchiolitis obliterans organizing pneumonia were seen. Perivascular infiltrates of inflammatory cells were seen multifocally within and adjacent to affected areas of the lung. There was also type II pneumocyte hyperplasia, alveolar oedema, presence fibrin with formation of hyaline membranes (de Wit et al., 2013).

\section{Diagnosis}

In Camels, IgG immunofluorescence assay, protein microarray and virus neutralisation assay could also be used in diagnosis (Perera et al., 2013; Haagmans et al., 2014; Ali et al., 2017). In humans, diagnosis could include the use of chest radiographs and computed tomographic scans (Arabi et al., 2014). Confirmation of MERS could be done by taking an intratracheal aspirate for detection test of MERSCoV RNA or taking both upper and lower respiratory tract specimens, such as sputum or bronchoscopic specimens (Lee et al., 2016). However, intratrachael aspirates are the best sample for detection of MERS-CoV. Blood specimen can also be tested in order to increase test sensitivity (Lee $e t$ al., 2016). Sero-conversion of antibodies using acute-phase and recovery-phase serum is required to confirm this disease (Lee et al., 2016). MERS-CoV infection could also be confirmed by positive real-time reverse transcriptase polymerase chain reaction assays (Park et al., 2016).

\section{Prevention and control}

Amongst the important measures to control MERS$\mathrm{CoV}$ spread are strict regulation of camel movement, regular herd screening and isolation of infected camels, use of personal protective equipment by camel handlers and enforcing rules banning all consumption of unpasteurized camel milk and urine (Omrani et al., 2015). In case of MERS-CoV infection in humans, counter-measures such as quarantining of persons including health workers who had contact with the infected patients (Park et al., 2016). In healthcare settings, control measures such as appropriate use of personal protective equipment, disinfection of the premises, and complete or partial closure of the affected wards and quarantine of exposed patients (Park et al., 2016). 


\section{Conclusions}

Middle East respiratory syndrome (MERS) is an emerging infectious disease. This zoonotic disease has mainly been reported in the Middle East. Nevertheless travel-associated cases and outbreaks have also been reported in addition to the nosocomial dimensions of the spread this disease. There are also reports of new lineages of this virus in areas such as in Africa where this virus and its effects has not been well reported. Therefore, this review article outlines current information about the aetiology, epidemiology, host risk factors, clinicopathological findings, diagnosis, prevention and control of this emerging disease.

\section{Conflict of Interest}

The authors declare that there are no conflicts of interest related to this article.

\section{References}

Ali M, El-Shesheny R, Kandeil A, Shehata M, Elsokary B, Gomaa M, ... Oludayo FF (2017). Cross-sectional surveillance of Middle East respiratory syndrome coronavirus (MERS-CoV) in dromedary camels and other mammals in Egypt, August 2015 to January 2016. Eurosurveillance 22(11).

Alraddadi BM, Watson JT, Almarashi A, Abedi GR, Turkistani, A., Sadran M, Housa A, Swerdlow DL (2016). Risk factors for primary Middle East Respiratory Syndrome coronavirus illness in humans, Saudi Arabia, 2014. Emerging Infectious Diseases 22(1):49-55.

Al-TawfiqJA, Memish ZA (2016). Drivers of MERS-CoV transmission: what do we know? Expert Review of Respiratory Medicine 10(3):331338.

Annan A, Baldwin HJ, Corman VM, KloseSM, Owusu M, NkrumahEE (2013). Human betacoronavirus 2cEMC/2012-related viruses in bats, Ghana and Europe.EmergingInfectious Diseases 19(3):456-459.

Arabi YM, Arifi AA, Balkhy HH, Najm H, Aldawood AS, Ghabashi A, ... Raiy B (2014). Clinical course and outcomes of critically ill patients with Middle East respiratory syndrome coronavirus infection. Annals of Internal Medicine 160(6):389-397.

Arabi YM, Harthi, A, Hussein J, Bouchama A, Johani S. Hajeer AH, ... Balkhy H (2015). Severe neurologic syndrome associated with Middle East respiratory syndrome corona virus (MERS-CoV). Infection 43(4):495-501.

Arabi YM, Balkhy HH, Hayden FG, Bouchama A, Luke T, Baillie JK, ... Fowler RA (2017). Middle East Respiratory Syndrome: special report. TheNewEngland Journal of Medicine 376(6):584594.

Azhar EI, El-Kafrawy SA, Farraj SA, Hassan AM, Al-Saeed M, Hashem AM, Madani TA (2014). Evidence for camel to human transmission of MERS coronavirus. The New England Journal of Medicine 370(26):2499-2505.

Bin SY, Heo JY, Song MS, Lee J, Kim EH, Park SJ, ... Choi, YK (2016). Environmental contamination and viral shedding in MERS patients during MERS-CoV outbreak in South Korea. Clinical Infectious Diseases 62(6):755-760.
Cotton M, Watson SJ, Kellam P, Al-Rabeeah AA, Makhdoom HQ, Assiri A (2013). Transmission and evolution of the Middle East respiratory syndrome coronavirus in Saudi Arabia: a descriptive genomic study. Lancet 382(9909):1993-2002.

De Groot RJ, Baker SC, Baric RS, Brown CS, Drosten C, Enjuanes L (2013). Middle East Respiratory Syndrome Coronavirus (MERS$\mathrm{CoV})$ : announcement of the Coronavirus study group.Journal of Virology 87(14):7790-7792.

de WitE, Rasmussen AL, Falzarano D, Bushmaker T, Feldmann F, Brining DL, ... Munster VJ (2013). Middle East Respiratory Syndrome Coronavirus (MERSCoV) causes transient lower respiratory tract infection in rhesus macaques. PNAS 110(41):16598-16603.

Haagmans BL, Al DhahirySH, Reusken CB, Raj VS, Galiano M, Myers R, ... Koopmans MP (2014). Middle East Respiratory Syndrome Coronavirus in dromedary camels: an outbreak investigation. The Lancet Infectious Diseases 14(2):140-145.

Haagmans BL, van den Brand JM, Provacia LB, Raj VS, Stittelaar KJ, Getu $\mathrm{S}, \ldots$ Osterhaus $\mathrm{AD}$ (2015). Asymptomatic middle east respiratory syndrome coronavirus infection in rabbits. Journal of Virology 89(11):6131-6135.

Haagmans BL, van den Brand JM, Provacia LB, Raj VS, Stittelaar KJ, Getu S, ... Fouchier RA (2015). Asymptomatic Middle East respiratory syndrome coronavirus infection in rabbits. Journal of Virology 89(11):6131-6135.

Hilgenfeld R, Peiris M (2013). From SARS to MERS: 10 years of research on highly pathogenic human coronaviruses. Antiviral Research 100(1):286-295.

Ithete NL, Stoffberg S, Corman VM, Cottontail VM, Richards LR, Schoeman MC (2013). Close relative of human MiddleEast respiratory syndrome coronavirus in bat, South Africa. Emerging Infectious Disease 19(10):1697-1699.

Al-TawfiqJA, Hinedi K, Abbasi S, Babike M, Sunji A, Eltigani M (2017). Hematologic, hepatic, and renal function changes in hospitalized patients with Middle East Respiratory Syndrome coronavirus. International Journal of Laboratory Haematology 39(3):272-278.

Kim SH, Chang SY, Sung M, Park JH, Bin KH, Lee, H, Min JY (2016). Extensive viable Middle East respiratory syndrome(MERS) coronavirus contamination in air and surrounding environment in MERS isolation wards. Clinical Infectious Disease 63(3):363-369.

Korea Center for Disease Control and Prevention (2016). Corrigendum to "Middle East Respiratory Syndrome Coronavirus Outbreak in the Republic of Korea, 2015” [Volume 6, Issue 4, August 2015, 269-278]. Osong Public Health and Research Perspective 2017:138.

Lee H, Ki CS, Sung H, Kim S, Seong MW, Yong D, Kim JS, Kim JH (2016). Guidelines for the laboratory diagnosis of Middle East respiratory syndrome coronavirus in Korea. Infectious and Chemotherapy 48(1):61-69.

Memish ZA, Al-TawfiqJA, Assiri A, AlRabiah FA, Al Hajjar S, Albarrak A, ... Alsubaie $S$ (2014). Middle East respiratory syndrome coronavirus disease is rare in children. World Journal of Clinical Pediatrics 5(4):391.

Memish ZA, Zumla AI, Al-Hakeem RF, Al-Rabeeah AA, Stephens GM (2013). Family cluster of Middle East respiratory syndrome coronavirus infections. The New England Journal of Medicine 368:2487-2494. 
324

Memish ZA, Mishra N, Olival KJ, FagboSF, Kapoor V, Epstein JH (2013). Middle East respiratory syndrome coronavirus in bats, Saudi Arabia. Emerging Infectious Diseases 19(11):1819-1823.

Mohd HA, Al-Tawfiq JA, Memish ZA (2016). Middle East respiratory syndrome coronavirus (MERS-CoV) origin and animal reservoir. Virology Journal 13(1):87.

Muller MA, Meyer B, Corman VM, Al-Masri M, Turkestani A, Ritz D, ... Memish ZA (2015). Presence of Middle East respiratory syndrome coronavirus antibodies in Saudi Arabia: a nationwide, cross-sectional, serological study. The Lancet Infectious Diseases 15(5):559-564.

Ng DL, Al Hosani F, Keating MK, Gerber SI, Jones TL, Metcalfe MG, ... Zaki SR (2016). Clinicopathologic, immunohistochemical, and ultrastructural findings of a fatal case of Middle East respiratory syndrome coronavirus infection in the United Arab Emirates, April 2014. The American Journal of Pathology 186(3):652-658.

Oboho IK, Tomczyk SM, Al-Asmari AM, Banjar AA, Al-Mugti H, Aloraini MS (2015) MERSCoV outbreak in Jeddah - a link to health care facilities. The New England Journal of Medicine 372(9):846-854.

Omrani AS, Al-Tawfiq JA, Memish ZA (2015). Middle East respiratory syndrome coronavirus (MERS-CoV): animal to human interaction. Pathogens and Global Health 109 (8):354362.

Park GE, Kang CI, Ko JH, Cho SY, Ha YE, Kim YJ, Chung DR (2017). Differential cell count and crp level in blood as predictors for Middle East respiratory syndrome coronavirus infection in acute febrile patients during nosocomial outbreak. Journal of Korean Medical Science 32(1):151-154.

Park SH, Kim WJ, Yoo J, Cho J (2016). Epidemiologic parameters of the Middle East respiratory syndrome outbreak in Korea, 2015. Infection and Chemotherapy 48(2):108-117.
Payne DC, Iblan I, Alqasrawi S, Al Nsour M, Rha B, Tohme RA, ... Al Abdallat MM (2014).Jordan MERS-CoV investigation team. Stillbirth during infection with Middle East respiratory syndrome coronavirus. Journal of Infectious Disease 209(12):870-1872.

Perera RA, Wang P, Gomaa MR, El-Shesheny R, Kandeil A, Bagato O, ... Kayali G (2013). Seroepidemiology for MERS coronavirus using microneutralisation and pseudoparticle virus neutralisation assays reveal a high prevalence of antibody in dromedary camels in Egypt, June 2013. Eurosurveillance 18(36):20574.

van Boheemen, S, de Graaf, M. Lauber C, Bestebroer TM, Raj VS, Zaki AM, ... Fouchier, RAM (2012). Genomic characterization of a newly discovered coronavirus associated with acute respiratory distress syndrome in humans. MBio 3(6):e00473-12.

Van Doremalen N, Munster VJ (2015). Animal models of Middle East respiratory syndrome coronavirus infection. Antiviral Research 122:2838.

World Health Organization (2015). Middle East respiratory syndrome coronavirus (MERS-CoV). Retrieved 2018 August 8 from http://www.who.int/ emergencies/mers-cov/en/.

Younan M, Bornstein S, Gluecks IV (2016). MERS and the dromedary camel trade between Africa and the Middle East. Tropical Animal Health and Production 48(6):1277-1282.

Zaki AM, van Boheemen S, Bestebroer TM, Osterhaus ADME, Fouchier RAM (2012). Isolation of a novel coronavirus from a man with pneumonia in Saudi Arabia. The New England Journal of Medicine 367(19):18141820. 